\title{
Probing superheavy dark matter with gravitational waves
}

\author{
Ligong Bian, ${ }^{a, b}$ Xuewen Liu ${ }^{c}$ and Ke-Pan Xie ${ }^{d, e, 1}$ \\ ${ }^{a}$ Department of Physics, Chongqing University, \\ Chongqing 401331, China \\ ${ }^{b}$ Chongqing Key Laboratory for Strongly Coupled Physics, \\ Chongqing 401331, China \\ ${ }^{c}$ Department of Physics, Yantai University, \\ Yantai 264005, China \\ ${ }^{d}$ Center for Theoretical Physics, Department of Physics and Astronomy, \\ Seoul National University, \\ Seoul 08826, South Korea \\ ${ }^{e}$ Department of Physics and Astronomy, University of Nebraska, \\ Lincoln, NE 68588, U.S.A. \\ E-mail: lgbycl@cqu.edu.cn, xuewenliu@ytu.edu.cn, kepan.xie@unl.edu
}

ABstract: We study the superheavy dark matter (DM) scenario in an extended $B-L$ model, where one generation of right-handed neutrino $\nu_{R}$ is the DM candidate. If there is a new lighter sterile neutrino that co-annihilate with the DM candidate, then the annihilation rate is exponentially enhanced, allowing a DM mass much heavier than the GriestKamionkowski bound $\left(\sim 10^{5} \mathrm{GeV}\right)$. We demonstrate that a DM mass $M_{\nu_{R}} \gtrsim 10^{13} \mathrm{GeV}$ can be achieved. Although beyond the scale of any traditional DM searching strategy, this scenario is testable via gravitational waves (GWs) emitted by the cosmic strings from the $\mathrm{U}(1)_{B-L}$ breaking. Quantitative calculations show that the DM mass $\mathcal{O}\left(10^{9}-10^{13} \mathrm{GeV}\right)$ can be probed by future GW detectors.

KeYwords: Beyond Standard Model, Cosmology of Theories beyond the SM

ARXIV EPRINT: 2107.13112

\footnotetext{
${ }^{1}$ Corresponding author.
} 


\section{Contents}

1 Introduction 1

2 The extended $B-L$ model 2

3 The superheavy $\nu_{R}$ dark matter 4

3.1 Thermal freeze-out 4

$\begin{array}{ll}3.2 & \text { Decay of } \psi \text { and the dark matter relic abundance }\end{array}$

$\begin{array}{lll}4 & \text { Cosmic strings and the gravitational wave signals } & 7\end{array}$

5 Conclusion 11

$\begin{array}{ll}\text { A The interaction rates } & 11\end{array}$

\section{Introduction}

The freeze-out of weakly interacting massive particles (WIMPs) [1] has been the most popular explanation for the particle origin of dark matter (DM) for decades. In this paradigm, the DM relic abundance is determined by [2-4]

$$
\Omega_{\mathrm{DM}} h^{2} \sim 0.1 \times \frac{1 \mathrm{pb}}{\left\langle\sigma_{\mathrm{ann} .} v\right\rangle} \sim 0.1 \times\left(\frac{0.01}{\alpha_{\mathrm{DM}}}\right)^{2}\left(\frac{M_{\mathrm{DM}}}{100 \mathrm{GeV}}\right)^{2},
$$

with $\sigma_{\text {ann. }}$ being the pair annihilation cross section of DM particles to the Standard Model (SM) particles, $v$ the relative velocity, and $\langle\ldots\rangle$ the thermal average. In the second approximate equality we have used $\left\langle\sigma_{\text {ann. }} v\right\rangle \sim \alpha_{\mathrm{DM}}^{2} / M_{\mathrm{DM}}^{2}$ where $\alpha_{\mathrm{DM}}$ is the finite structure constant of the coupling between the dark and SM sectors. Eq. (1.1) shows that if the dark matter is at electroweak (EW) scale and its coupling is of the order of the EW coupling, then the freeze-out mechanism can explain the observed DM density $\Omega_{\mathrm{DM}} h^{2} \approx 0.12[5,6]$. This is the so-called "WIMP miracle", which has motivated enormous efforts to search for EW scale WIMPs via direct [7], indirect [8] and collider [9] experiments.

However, WIMP mass deviating from EW scale is possible. For example if $\alpha_{\mathrm{DM}} \gg 0.01$, then $M_{\mathrm{DM}} \gg 100 \mathrm{GeV}$ can yield the correct DM abundance. Since $\alpha_{\mathrm{DM}}$ has an upper limit $\sim 4 \pi$ set by the unitarity bound, $M_{\mathrm{DM}}$ also has an upper limit, which is $\sim 300 \mathrm{TeV}$ derived from the partial wave analysis, known as the Griest-Kamionkowski (GK) bound [10]. ${ }^{1}$ It is known that DM can be heavier than the GK bound in case of non-thermal dynamics, nonstandard cosmological history [11-28] or the first-order cosmic phase transition [29-32].

\footnotetext{
${ }^{1}$ This bound applies to the elementary particle DM. If the DM is a composite object, another bound $R_{\mathrm{DM}} \gtrsim 7.5 \times 10^{-7} \mathrm{fm}$ applies to the DM size [10].
} 
However, as proposed in refs. [33, 34], DM mass beyond the GK bound is also possible within the thermal freeze-out framework, as long as there is a lighter unstable species that co-annihilates with the DM candidate (dubbed as the "zombie collision" [34]) and exponentially enhances the interaction rate. In other words, for the same coupling, such an annihilation allows an exponentially heavier DM mass compared to the normal DM pair annihilation scenario. The extra entropy produced from the late time decay of the lighter species further dilutes the DM density, leading to an even higher DM mass upper limit that can reach $10^{16} \mathrm{GeV}[33]$.

While the above zombie annihilation mechanism is theoretically appealing, it is very challenging to probe such superheavy DM via the traditional direct, indirect or collider experiments. In this article, we propose a zombie annihilation mechanism that is associated with the breaking of a U(1) symmetry, which leads to the formation of cosmic strings that can be detected via the gravitational wave (GW) signals at current or future GW detectors. As a benchmark, we study an extended $B-L$ model, where one generation of the righthanded neutrino (RHN) is the DM candidate, and a new Dirac sterile neutrino serves as the lighter species for co-annihilation. Section 2 introduces the model, while section 3 is devoted to the calculation of thermal freeze-out and DM relic abundance. We then investigate the corresponding cosmic strings and GW signals in section 4, where the correlation to the DM scenario is obtained, and the recent NANOGrav excess is also commented. The conclusion will be given in section 5 .

\section{The extended $B-L$ model}

We begin with the $B-L$ model [35-38], which gauges the $\mathrm{U}(1)_{B-L}$ group, with $B$ and $L$ being the baryon and lepton number, respectively. In the $B-L$ model, three generations of Majorana RHNs $\nu_{R}^{i}$ (with $B-L=-1$ ) are introduced for gauge anomaly cancellation. The new gauge boson of $\mathrm{U}(1)_{B-L}$ is denoted as $Z^{\prime}$, and a complex scalar field $\Phi$ (with $B-L=2)$ is introduced to break the $\mathrm{U}(1)_{B-L}$ and provide mass for $Z^{\prime}$. The relevant Lagrangian reads

$$
\begin{aligned}
\mathcal{L}_{B-L}= & \sum_{i} \bar{\nu}_{R}^{i} i \not D \nu_{R}^{i}-\frac{1}{2} \sum_{i, j}\left(\lambda_{R}^{i j} \bar{\nu}_{R}^{i, c} \Phi \nu_{R}^{j}+\text { h.c. }\right)-\sum_{i, j}\left(\lambda_{D}^{i j} \bar{\ell}_{L}^{i} \tilde{H} \nu_{R}^{j}+\text { h.c. }\right) \\
& +D_{\mu} \Phi^{\dagger} D^{\mu} \Phi-\lambda_{\phi}\left(|\Phi|^{2}-\frac{v_{\phi}^{2}}{2}\right)^{2}-\frac{1}{4} Z_{\mu \nu}^{\prime} Z^{\prime \mu \nu}
\end{aligned}
$$

where $i, j$ are generation indices and $D_{\mu}=\partial_{\mu}-i g_{B-L} X Z_{\mu}^{\prime}$ is the gauge covariant derivative with $X$ being the corresponding $B-L$ number. The scalar potential triggers a spontaneous symmetry breaking at $\langle|\Phi|\rangle=v_{\phi} / \sqrt{2}$. If we parametrize $\Phi$ as $(\phi+i \eta) / \sqrt{2}$, then after the $\mathrm{U}(1)_{B-L}$ breaking $\eta$ is absorbed as the longitudinal mode of $Z^{\prime}$, and the particles obtain the following masses

$$
M_{Z^{\prime}}=2 g_{B-L} v_{\phi}, \quad M_{\nu_{R}}^{i j}=\lambda_{R}^{i j} \frac{v_{\phi}}{\sqrt{2}}, \quad M_{\phi}=\sqrt{2 \lambda_{\phi}} v_{\phi}
$$




\begin{tabular}{|c|c|c|c|c|c|}
\hline & $\nu_{R}^{1,2}$ & $\nu_{R}^{3}$ & $\Phi$ & $\psi$ & $S$ \\
\hline$B-L$ & -1 & -1 & 2 & -1 & 0 \\
\hline $\mathbb{Z}_{2}$ & 1 & -1 & 1 & -1 & 1 \\
\hline
\end{tabular}

Table 1. Quantum numbers of the BSM particles. All particles listed here are singlets under the SM gauge group.

The Lagrangian (2.1) can elegantly explain the extremely small mass of the SM neutrinos and the matter-antimatter asymmetry of the Universe via Type-I seesaw [39] and leptogenesis [40], respectively. Especially, the explanation of neutrino mass $\sim 0.1 \mathrm{eV}[5,41]$ prefers superheavy RHNs, since the seesaw mechanism requires $M_{\nu_{R}} \sim \lambda_{D} \lambda_{D}^{\dagger} \times 10^{14} \mathrm{GeV}$.

For the sake of a DM candidate, we adjust eq. (2.1) by assigning a $\mathbb{Z}_{2}$ symmetry, under which the third generation RHN is odd while all other particles are even. Consequently, $\lambda_{D}^{i 3}=0$ for $i=1,2,3$ and $\lambda_{R}^{j 3}=0$ for $j=1,2$, making $\nu_{R}^{3}$ free from the $\nu_{R}^{3} \rightarrow \ell H$ decay and hence can be a DM candidate. This setup is generally adopted in the $\nu_{R}$-DM scenarios $[42-47] .^{2}$ To realize the zombie co-annihilation, we further extend the model by introducing a Dirac sterile neutrino $\psi$ (with $B-L=-1$ ) and a gauge singlet real scalar mediator $S .^{3}$ The quantum numbers of the particles beyond the SM (BSM) are summarized in table 1 . Since hereafter we only discuss the DM candidate $\nu_{R}^{3}$, for simplicity we will denote $\nu_{R}^{3}$ as $\nu_{R}$ and $\lambda_{R}^{33}$ as $\lambda_{R}$. The Lagrangian relevant for DM reads

$$
\mathcal{L}_{\mathrm{DM}}=\bar{\psi}\left(i \not D-M_{\psi}\right) \psi+\frac{1}{2} \partial_{\mu} S \partial^{\mu} S-\frac{1}{2} M_{S}^{2} S^{2}+\left(\lambda_{1} S \bar{\psi} \nu_{R}+\text { h.c. }\right)+\lambda_{2} S \bar{\psi} \psi,
$$

which provides the zombie collisions

$$
\nu_{R}+\psi \rightarrow \psi+\psi, \quad \nu_{R}+\psi \rightarrow \psi+\bar{\psi}
$$

and their charge conjugations via the exchange of an $S$ mediator. If we name $\nu_{R}$ as a "survivor" and $\psi$ as a "zombie", then eq. (2.4) is infecting a survivor to a zombie, and that is why it is called a zombie collison [34]. As we will see in the next section, for $M_{\psi}<M_{\nu_{R}}=\lambda_{R} v_{\phi} / \sqrt{2}$, the annihilation rate is exponentially enhanced and $M_{\nu_{R}}$ can reach $10^{13} \mathrm{GeV}$ while still generating the correct DM relic abundance.

We work in the parameter space

$$
M_{\nu_{R}}+M_{\psi}<M_{S}, \quad M_{\psi}<M_{\nu_{R}}<3 M_{\psi},
$$

so that the DM decay channels $\nu_{R} \rightarrow \psi S / \bar{\psi} S$ or $\nu_{R} \rightarrow \psi \psi \bar{\psi}$ are kinematically forbidden, while the mediator $S$ can decay to pairs of $\bar{\psi} \nu_{R}, \psi \nu_{R}$, and $\psi \bar{\psi}$, with the width

$$
\Gamma_{S}=\Gamma_{S \rightarrow \bar{\psi} \nu_{R}}+\Gamma_{S \rightarrow \psi \nu_{R}}+\Gamma_{S \rightarrow \psi \bar{\psi}}
$$

\footnotetext{
${ }^{2}$ Two generations of $\mathbb{Z}_{2}$-even RHNs are already sufficient to explain the neutrino oscillation data and realize leptogenesis, see the recent review [48] and the references therein.

${ }^{3}$ See ref. [49] for the low-scale phase transition study in the complex singlet extended $U(1)_{B-L}$ model.
} 
where

$$
\begin{aligned}
\Gamma_{S \rightarrow \bar{\psi} \nu_{R}}=\Gamma_{S \rightarrow \bar{\psi} \nu_{R}} & =\frac{\lambda_{1}^{2}}{16 \pi} M_{S}\left(1-a_{R}-a_{\psi}\right) \sqrt{1-2\left(a_{R}+a_{\psi}\right)+\left(a_{R}-a_{\psi}\right)^{2}}, \\
\Gamma_{S \rightarrow \psi \bar{\psi}} & =\frac{\lambda_{2}^{2}}{8 \pi} M_{S}\left(1-4 a_{\psi}\right)^{3 / 2},
\end{aligned}
$$

and $a_{R} \equiv M_{\nu_{R}}^{2} / M_{S}^{2}, a_{\psi} \equiv M_{\psi}^{2} / M_{S}^{2}$.

The final essential ingredient of the zombie mechanism is an appropriate decay channel for the Dirac sterile neutrino $\psi$. After the freeze-out of $\nu_{R}, \psi$ needs to decay into the SM particles, otherwise itself is a WIMP DM candidate that satisfies the GK bound, and hence $\nu_{R}$ cannot exceed the GK bound due to the $M_{\nu_{R}}<3 M_{\psi}$ constraint. Since $\psi$ is odd under the $\mathbb{Z}_{2}$ while the SM particles are even, such $\psi$ decay must involve some $\mathbb{Z}_{2}$-breaking interactions, which can also result in the decay of the DM candidate $\nu_{R}$. To make $\nu_{R}$ sufficiently long-lived (with a lifetime longer than $10^{27} \mathrm{~s}$, as required by the diffuse gammaray spectrum [50-52]), the breaking of $\mathbb{Z}_{2}$ should be mediated by either extremely small couplings or extremely high scales with some level of fine-tuning. ${ }^{4}$ For the former case, we can have an interacting vertex like $y_{\psi} \bar{\ell}_{L} H \psi_{R}$ with $\left|y_{\psi}\right| \ll 1$; while for the latter case, a dimension- 6 operator can be induced by exchanging a new heavy color triplet scalar

$$
\mathcal{L}_{6}=\frac{1}{\Lambda^{2}} \sum_{i, j, j^{\prime}}\left(\bar{\psi}^{c} u_{R}^{i}\right)\left(\bar{d}_{R}^{j, c} d_{R}^{j^{\prime}}\right)+\text { h.c. },
$$

where $i, j$ and $j^{\prime}$ are generation indices and $j \neq j^{\prime}$. The color indices of quarks are implicitly summed up in a completely asymmetric way via the Levi-Civita symbol to yield a color singlet. We will take eq. (2.8) as an example for the further study, but keep in mind that the zombie DM mechanism holds as long as $\psi$ can decay via a tiny $\mathbb{Z}_{2}$-breaking interaction. According to eq. (2.8), the decay width of $\psi$ is

$$
\Gamma_{\psi}=\Gamma_{\psi \rightarrow 3 j}=\frac{1}{1024 \pi^{3}} \frac{M_{\psi}^{5}}{\Lambda^{4}},
$$

assuming an exclusive $u d s$-quark final state. Note that eq. (2.8) also allows the decay $\nu_{R} \rightarrow \psi \psi^{(*)} \bar{\psi}^{(*)} \rightarrow 9 j$ via one or two off-shell $\psi$ 's, and hence a high $\Lambda$ is required to keep $\nu_{R}$ sufficiently long-lived. On the other hand, the same high $\Lambda$ also results in the late time decay of $\psi$, which can produce entropy to dilute the $\nu_{R}$ abundance.

\section{The superheavy $\nu_{R}$ dark matter}

\subsection{Thermal freeze-out}

Assume the reheating temperature after inflation is higher than $M_{\nu_{R}}$ and hence both $\nu_{R}$ and $\psi$ are originally in thermal equilibrium with each other and the SM particles, and the number densities are described by

$$
n_{\alpha}^{\mathrm{eq}}=2 \int \frac{d^{3} p}{(2 \pi)^{3}} e^{-E_{\alpha} / T}=2 \times \frac{M_{\alpha}^{2} T}{2 \pi^{2}} K_{2}\left(\frac{M_{\alpha}}{T}\right),
$$

\footnotetext{
${ }^{4}$ The word "fine-tuning" is also in the sense that we assume only $\psi$ directly participates in the $\mathbb{Z}_{2}$-breaking interactions.
} 
where $\alpha=\nu_{R}, \psi$ or $\bar{\psi}, K_{2}$ is the modified Bessel function of the second kind, and the factor 2 is for spin degeneracy. As the Universe expands and cools down, the reaction rate between $\nu_{R}$ and $\psi$ (see eq. (2.4)) drops. When the interaction rate is lower than the Universe expansion rate, $\nu_{R}$ deviates from the chemical equilibrium and eventually freeze-out to be the DM candidate. The freeze-out process can be characterized by a set of Boltzmann equations, which are discussed in detail below.

Consider the radiation dominated era that the energy and entropy densities are respectively

$$
\rho=\frac{\pi^{2}}{30} g_{*} T^{4}, \quad s=\frac{2 \pi^{2}}{45} g_{*} T^{3},
$$

with $g_{*}$ being the number of relativistic degrees of freedom which we take to be the SM value 106.75. The Hubble constant can be solved as

$$
H=\left(\frac{8 \pi}{3 M_{\mathrm{Pl}}^{2}} \frac{\pi^{2}}{30} g_{*} T^{4}\right)^{1 / 2}=2 \pi \sqrt{\frac{\pi g_{*}}{45}} \frac{T^{2}}{M_{\mathrm{Pl}}},
$$

with $M_{\mathrm{Pl}}=1.22 \times 10^{19} \mathrm{GeV}$ the Planck scale. Define the dimensionless parameter $z=$ $M_{\nu_{R}} / T$, then $s=s_{\nu_{R}} / z^{3}$ and $H=H_{\nu_{R}} / z^{2}$, where

$$
\left.s_{\nu_{R}} \equiv s\right|_{T=M_{\nu_{R}}},\left.\quad H_{\nu_{R}} \equiv H\right|_{T=M_{\nu_{R}}} .
$$

Finally, we define the particle abundance as $Y_{\alpha}=n_{\alpha} / s$, i.e. the ratio of number density to the entropy density, and hence the abundances of the equilibrium distributions are

$$
Y_{\nu_{R}}^{\mathrm{eq}}=\frac{45 z^{2}}{2 \pi^{4} g_{*}} K_{2}(z), \quad Y_{\psi}^{\mathrm{eq}}=\frac{45 z^{2}}{2 \pi^{4} g_{*}}\left(\frac{M_{\psi}}{M_{\nu_{R}}}\right)^{2} K_{2}\left(\frac{M_{\psi}}{M_{\nu_{R}}} z\right) .
$$

Under above conventions, the Boltzmann equations of $\nu_{R}$ and $\psi$ can be expressed as a set of ordinary differential equations,

$$
\begin{aligned}
\frac{s_{\nu_{R}} H_{\nu_{R}}}{z^{4}} \frac{d Y_{\nu_{R}}}{d z}= & -\frac{Y_{\psi}}{Y_{\psi}^{\mathrm{eq}}}\left(\frac{Y_{\nu_{R}}}{Y_{\nu_{R}}^{\mathrm{eq}}}-\frac{Y_{\psi}}{Y_{\psi}^{\mathrm{eq}}}\right)\left(2 \gamma_{\nu_{R} \psi \rightarrow \psi \psi}+2 \gamma_{\nu_{R} \bar{\psi} \rightarrow \psi \bar{\psi}}\right) \\
& -\left(\frac{Y_{\nu_{R}}^{2}}{\left(Y_{\nu_{R}}^{\mathrm{eq}}\right)^{2}}-\frac{Y_{\psi}^{2}}{\left(Y_{\psi}^{\mathrm{eq}}\right)^{2}}\right) 2\left(\gamma_{\nu_{R} \nu_{R} \rightarrow \psi \bar{\psi}}+2 \gamma_{\nu_{R} \nu_{R} \rightarrow \psi \psi}\right)-\left(\frac{Y_{\nu_{R}}^{2}}{\left(Y_{\nu_{R}}^{\mathrm{eq}}\right)^{2}}-1\right) 2 \gamma_{\nu_{R} \nu_{R} \rightarrow f \bar{f}}, \\
\frac{s_{\nu_{R}} H_{\nu_{R}}}{z^{4}} \frac{d Y_{\psi}}{d z}= & \frac{Y_{\psi}}{Y_{\psi}^{\mathrm{eq}}}\left(\frac{Y_{\nu_{R}}}{Y_{\nu_{R}}^{\mathrm{eq}}}-\frac{Y_{\psi}}{Y_{\psi}^{\mathrm{eq}}}\right)\left(\gamma_{\nu_{R} \psi \rightarrow \psi \psi}+\gamma_{\nu_{R} \bar{\psi} \rightarrow \psi \bar{\psi}}\right) \\
& +\left(\frac{Y_{\nu_{R}}^{2}}{\left(Y_{\nu_{R}}^{\mathrm{eq}}\right)^{2}}-\frac{Y_{\psi}^{2}}{\left(Y_{\psi}^{\mathrm{eq}}\right)^{2}}\right)\left(\gamma_{\nu_{R} \nu_{R} \rightarrow \psi \bar{\psi}}+2 \gamma_{\nu_{R} \nu_{R} \rightarrow \psi \psi}\right)-\left(\frac{Y_{\psi}^{2}}{\left(Y_{\psi}^{\mathrm{eq}}\right)^{2}}-1\right) \gamma_{\psi \bar{\psi} \rightarrow f \bar{f}},
\end{aligned}
$$

and $Y_{\nu_{R}}^{\infty}=Y_{\nu_{R}}(z \rightarrow \infty)$ is the freeze-out relic abundance of the DM candidate $\nu_{R}$. Here the $\gamma_{a b \rightarrow c d}$ 's are the corresponding interaction rates,

$$
\gamma_{a b \rightarrow c d}=n_{a}^{\mathrm{eq}} n_{b}^{\mathrm{eq}}\left\langle\sigma_{a b \rightarrow c d} v\right\rangle,
$$

and the detailed definitions can be found in appendix A. Since $M_{\nu_{R}}>M_{\psi}$, the abundance of $\psi$ is higher than $\nu_{R}$, i.e. $n_{\psi}^{\mathrm{eq}} \sim\left(n_{\nu_{R}}^{\mathrm{eq}}\right)^{M_{\nu_{R}} / M_{\psi}}$, leading to an exponentially enhanced 
annihilation rate compared with the WIMP scenario. The factor "2" in front of $\gamma_{\nu_{R} \psi \rightarrow \psi \psi}$, $\gamma_{\nu_{R} \bar{\psi} \rightarrow \psi \bar{\psi}}$ and $\gamma_{\nu_{R} \nu_{R} \rightarrow \psi \psi}$ comes from charge conjugation (e.g. $\gamma_{\nu_{R} \bar{\psi} \rightarrow \bar{\psi} \bar{\psi}}$ ), and we have made use of $Y_{\psi} \equiv Y_{\bar{\psi}}$ due to CP conservation. For simplicity, we assume $M_{\phi}, M_{Z^{\prime}} \gtrsim M_{\nu_{R}}$ so that the $B-L$ scalar $\phi$ and gauge boson $Z^{\prime}$ do not participate in the Boltzmann equations explicitly, but $Z^{\prime}$ contribute to $\gamma_{\nu_{R} \nu_{R} \rightarrow f \bar{f}}$ and $\gamma_{\psi \bar{\psi} \rightarrow f \bar{f}}$ (where $f$ denotes the SM fermions in equilibrium) via the off-shell $s$-channel diagrams. In principle, the decay $\psi \rightarrow j j j$ and scattering $\psi j \rightarrow j j$ induced by the dimension-6 operator in eq. (2.8) also affect the evolution of $Y_{\psi}$, but the effect is completely negligible due to the large $\Lambda$. Instead, the late time $\psi$ decay after $\nu_{R}$ freeze-out plays an important role, as discussed in the next subsection.

\subsection{Decay of $\psi$ and the dark matter relic abundance}

Due to its long life time, $\psi$ can be treated as a stable particle during the freeze-out of $\nu_{R}$, and its "relic abundance" is given by $Y_{\psi}^{\infty}=Y_{\psi}(z \rightarrow \infty)$. After freeze-out, the energy density of $\psi$ scales as $a^{-3}$ where $a(t)$ is the Friedmann-Lemaitre-Robertson-Walker (FLRW) scale factor, while the radiation energy density scales as $a^{-4}$. As a result, if $\psi$ is sufficiently long-lived, it dominates the Universe energy at the late time, and its decay would generate significant entropy that further suppresses the $\nu_{R}$ relic abundance. The dilution factor can be estimated quickly by the following considerations: $\psi$ decays at $T=T_{\psi}$ when $\Gamma_{\psi} \sim H$, i.e.

$$
\left.\Gamma_{\psi}^{2} \sim H^{2}\right|_{T=T_{\psi}} \approx \frac{8 \pi}{3 M_{\mathrm{Pl}}^{2}} M_{\psi} Y_{\psi}^{\infty}\left(\frac{2 \pi^{2}}{45} g_{*} T_{\psi}^{3}\right),
$$

If $\psi$ decays to $j j j$ very promptly at $t_{\psi}=1 / \Gamma_{\psi}$ and reheats the Universe up to $T_{\psi}^{\prime}$, by energy conservation we have

$$
\frac{\pi^{2}}{30} g_{*} T_{\psi}^{\prime 4}=M_{\psi} Y_{\psi}^{\infty}\left(\frac{2 \pi^{2}}{45} g_{*} T_{\psi}^{3}\right) .
$$

Combining these two equations, one obtains the entropy enhancement factor (or equivalently, the DM density dilution factor)

$$
\Delta_{\psi}=\frac{S_{\text {after }}}{S_{\text {before }}} \approx\left(\frac{T_{\psi}^{\prime}}{T_{\psi}}\right)^{3} \sim g_{*}^{1 / 4} \frac{M_{\psi} Y_{\psi}^{\infty}}{\sqrt{M_{\mathrm{Pl}} \Gamma_{\psi}}},
$$

and the final DM relic abundance is $Y_{\mathrm{DM}}=Y_{\nu_{R}}^{\infty} / \Delta_{\psi}$. A more detailed treatment in ref. [53] yields

$$
\Delta_{\psi} \approx 1.83\left\langle g_{*}^{1 / 3}\right\rangle^{3 / 4} \frac{M_{\psi} Y_{\psi}^{\infty}}{\sqrt{M_{\mathrm{Pl}} \Gamma_{\psi}}},
$$

which will be adopted in our numerical study.

Now we are ready to calculate the DM relic abundance by solving the Boltzmann equations (3.6) and (3.7) to get $Y_{\nu_{R}}^{\infty}$, and combining the dilution factor $\Delta_{\psi}$. For the numerical study, we fix $g_{B-L}=1, \lambda_{R}=0.1, M_{\nu_{R}}=1.9 M_{\psi}, M_{S}=2 M_{\nu_{R}}$ and $\lambda_{1}=\lambda_{2}$, and vary $M_{\nu_{R}}, \lambda_{1}$ and $\Lambda$ to find four benchmark points (BPs) listed in table 2. All BPs can yield the correct relic abundance

$$
\Omega_{\mathrm{DM}} h^{2}=\frac{8 \pi h^{2}}{3 M_{\mathrm{Pl}}^{2} H_{0}^{2}} \frac{Y_{\nu_{R}}^{\infty} s_{0}}{\Delta_{\psi}} M_{\nu_{R}} \approx 0.12,
$$




\begin{tabular}{|c|c|c|c||c|c|c|c|}
\hline & $M_{\nu_{R}}$ & $\lambda_{1}$ & $\Lambda$ & $T_{\psi}$ & $T_{\psi}^{\prime}$ & $\Delta_{\psi}$ & $\tau_{\nu_{R}}$ \\
\hline BP1 & $1.5 \times 10^{13} \mathrm{GeV}$ & 1.5 & $2 \times 10^{18} \mathrm{GeV}$ & $3.0 \mathrm{GeV}$ & $2.0 \times 10^{3} \mathrm{GeV}$ & $3.1 \times 10^{8}$ & $1.0 \times 10^{28} \mathrm{~s}$ \\
\hline BP2 & $1.0 \times 10^{12} \mathrm{GeV}$ & 0.74 & $1 \times 10^{17} \mathrm{GeV}$ & $2.7 \mathrm{GeV}$ & $9.4 \times 10^{2} \mathrm{GeV}$ & $4.1 \times 10^{7}$ & $2.3 \times 10^{29} \mathrm{~s}$ \\
\hline BP3 & $4.0 \times 10^{10} \mathrm{GeV}$ & 0.40 & $2 \times 10^{15} \mathrm{GeV}$ & $7.7 \mathrm{GeV}$ & $7.5 \times 10^{2} \mathrm{GeV}$ & $9.1 \times 10^{5}$ & $2.5 \times 10^{29} \mathrm{~s}$ \\
\hline BP4 & $3.0 \times 10^{9} \mathrm{GeV}$ & 0.30 & $1 \times 10^{14} \mathrm{GeV}$ & $16 \mathrm{GeV}$ & $4.6 \times 10^{2} \mathrm{GeV}$ & $2.4 \times 10^{4}$ & $4.1 \times 10^{29} \mathrm{~s}$ \\
\hline
\end{tabular}

Table 2. The BPs for the superheavy RHN DM scenario. $g_{B-L}=1, \lambda_{R}=0.1, M_{\nu_{R}}=1.9 M_{\psi}$, $M_{S}=2 M_{\nu_{R}}$ and $\lambda_{1}=\lambda_{2}$ are fixed, and the first three columns $M_{\nu_{R}}$ (RHN mass), $\lambda_{1}\left(\nu_{R^{-}} \psi-S\right.$

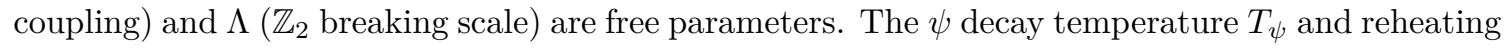
temperature $T_{\psi}^{\prime}$, dilution factor $\Delta_{\psi}$ and the DM life time $\tau_{\nu_{R}}$ are derived, see the text.

where $H_{0}=100 h \mathrm{~km} / \mathrm{s} / \mathrm{Mpc}$ (with $h=0.674$ ) and $s_{0}=2891.2 \mathrm{~cm}^{-3}$ are current Hubble constant and entropy density, respectively [6]. As we can see, due to the enhanced cross section from the light sterile neutrino (i.e. zombie collision) and the dilution factor from $\psi$ decay, a $1.5 \times 10^{13} \mathrm{GeV}$ RHN can still yield the correct DM abundance with a moderate coupling $\lambda_{1}=\lambda_{2}=1.5$. Even for a small coupling $\lambda_{1}=\lambda_{2}=0.30$, the RHN DM can be as heavy as $3.0 \times 10^{9} \mathrm{GeV}$, well above the GK bound. In our mass setup, $\nu_{R}$ can decay to 9 jets via 2 off-shell $\psi$ 's, and the life time is calculated by the FeynRules [54] and MadGraph5_aMC@NLO [55] packages. $\tau_{\nu_{R}} \gtrsim 10^{27} \mathrm{~s}$ is satisfied in all BPs, as required by the diffuse gamma-ray spectrum [50-52].

To distinguish and compare the contributions from "zombie collision" and " $\psi$ decay dilution" to the DM relic density, we plot the $Y_{\nu_{R}}$ evolution as blue curves in the BPs in figure 1. The equilibrium distribution $Y_{\nu_{R}}^{\text {eq }}$ is shown in green curves for reference, such that one can clearly see the $\nu_{R}$ deviates from the thermal equilibrium and freeze-out to a constant abundance $Y_{\nu_{R}}^{\infty}$. The late time decay of $\psi$ will dilute the freeze-out abundance to $Y_{\nu_{R}}^{\infty} / \Delta_{\psi}$, which is eventually $Y_{\mathrm{DM}} \approx 0.8 \mathrm{eV} / M_{\nu_{R}}$, as shown in red dashed straight lines. To manifest the importance of the zombie collision, we also plot the $Y_{\nu_{\mathrm{R}}}$ evolution assuming $\lambda_{1}=\lambda_{2}=0$ in orange curves. In that case, $\nu_{R}$ freeze-out in a very high abundance and hence cannot provide the correct DM density even with the help of the $\psi$ decay dilution. By comparing the curves of $Y_{\nu_{R}}, Y_{\nu_{R}}$ (without zombie) and $Y_{\mathrm{DM}}$, one can see that the contributions from zombie collision and $\psi$ decay dilution are comparable.

\section{Cosmic strings and the gravitational wave signals}

The BPs obtained in the last section are for $M_{\nu_{R}} \gtrsim 10^{9} \mathrm{GeV}$, much higher than the available energy scale of any traditional DM direct, indirect or collider searches, making it almost hopeless to test superheavy DM scenario. However, the recently developed GW astronomy offers an unique opportunity to probe this scenario: as the RHN mass $M_{\nu_{R}}$ is associated with a high-scale $\mathrm{U}(1)_{B-L}$ breaking, which forms cosmic strings that can generate detectable stochastic GW signals [56-62].

Cosmic strings are one dimensional topological defects form during a spontaneous symmetry breaking if the topology of the vacuum is not simply connected [63]. In our scenario, 

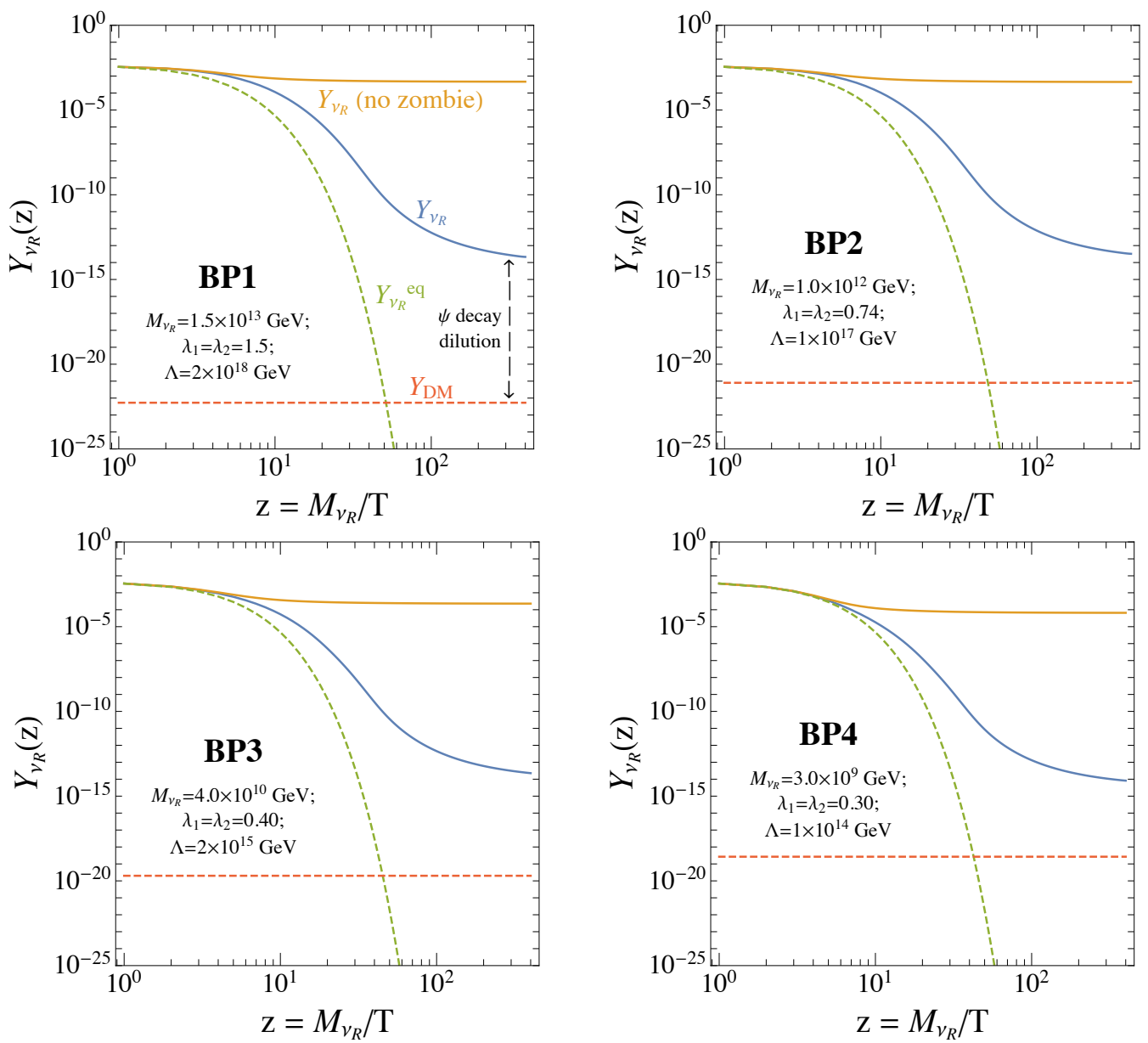

Figure 1. Evolution of the $\nu_{R}$ abundance for the four BPs in table 2. $Y_{\nu_{R}}$ and $Y_{\nu_{R}}^{\text {eq }}$ are shown in blue and green curves, respectively. For comparison, we also show in orange curves the $Y_{\nu_{R}}$ evolution without the zombie collision.

we consider Nambu-Goto cosmic strings that can form after the $\mathrm{U}(1)_{B-L}$ breaking, and the energy density per unit length $\mu \sim v_{\phi}^{2}$. A very important observable for the cosmic strings is the dimensionless combination

$$
G \mu \sim \frac{v_{\phi}^{2}}{M_{\mathrm{Pl}}^{2}} \sim 10^{-10} \times\left(\frac{M_{\nu_{R}} / \lambda_{R}}{10^{14} \mathrm{GeV}}\right)^{2}
$$

where $G$ is the Newton's constant of gravitation. After formation, the collisions and selfinteractions of strings produce sub-horizon, non-self-interacting string loops, which emit GWs via cusp, kink and kink-kink collisions, and they produce GWs throughout the Universe history. The incoherent superposition of such continuous emission results in today's stochastic GW signals.

According to above physical picture, the GW spectrum today can be expressed as

$$
\Omega_{\mathrm{GW}}(f) h^{2}=\frac{8 \pi h^{2}}{3 M_{\mathrm{Pl}}^{2} H_{0}^{2}} \int_{0}^{t_{0}} d t\left(\frac{a(t)}{a\left(t_{0}\right)}\right)^{3} \int_{0}^{\infty} d \ell n_{\mathrm{CS}}(\ell, t) P_{\mathrm{GW}}\left(\frac{a\left(t_{0}\right)}{a(t)} f, \ell\right)
$$


where $a(t)$ is the FLRW scale factor and $t_{0}$ is the current cosmic time. $n_{\mathrm{CS}}(\ell, t)$ is the number density of sub-horizon string loops with invariant length $\ell$ at cosmic time $t$, while $P_{\mathrm{GW}}(f, \ell)$ is the loop power spectrum describing the power of GW with frequency $f$ emitted from a loop with length $\ell$. We follow the method described in ref. [58] to calculate the GWs from the Nambu-Goto string [64] by transforming eq. (4.2) into [58, 65]

$$
\Omega_{\mathrm{GW}}(f) h^{2}=\frac{8 \pi h^{2}}{3 M_{\mathrm{Pl}}^{2} H_{0}^{2}} G \mu^{2} f \sum_{n=1}^{\infty} C_{n}(f) P_{n},
$$

where $n=1,2, \ldots$, labels the radiation frequencies $\omega_{n}=2 \pi n /(\ell / 2)$, and $P_{n}$ is the corresponding average loop power spectrum which we use the numerical results from ref. [65], and

$$
C_{n}=\frac{2 n}{f^{2}} \int_{0}^{\infty} \frac{d z}{H(z)(1+z)^{6}} n_{\mathrm{CS}}\left(\frac{2 n}{(1+z) f}, t(z)\right),
$$

where we have transformed the integral variable from time $t$ to the redshift $z$.

To evaluate the integration in eq. (4.4), the cosmic time and Hubble constant should be expressed as functions of redshift

$$
t(z)=\int_{z}^{\infty} \frac{d z^{\prime}}{H\left(z^{\prime}\right)\left(1+z^{\prime}\right)} ; \quad H(z)=H_{0} \sqrt{\Omega_{r} \mathcal{G}(z)(1+z)^{4}+\Omega_{m}(1+z)^{3}+\Omega_{\Lambda}},
$$

with the abundance [5]

$$
\Omega_{r}=9.1476 \times 10^{-5}, \quad \Omega_{m}=0.308, \quad \Omega_{\Lambda}=1-\Omega_{r}-\Omega_{m},
$$

and the function

$$
\mathcal{G}(z)=\frac{g_{*}(t)}{g_{*}\left(t_{0}\right)}\left(\frac{g_{S}\left(t_{0}\right)}{g_{S}(t)}\right)^{4 / 3} \approx\left\{\begin{array}{lc}
1, & z<10^{9} \\
0.83, & 10^{9}<z<2 \times 10^{12} \\
0.39, & z>2 \times 10^{12}
\end{array}\right.
$$

enfolds the change of relativistic degrees of freedom at $e^{+} e^{-}$annihilation $\left(200 \mathrm{keV}, z=10^{9}\right)$ and QCD phase transition $\left(200 \mathrm{MeV}, z=2 \times 10^{12}\right)$ [66]. The cosmic string number density is $[67]$

$$
n_{\mathrm{CS}}^{r}(\ell, t)=\frac{0.18}{t^{3 / 2}(\ell+\Gamma G \mu t)^{5 / 2}}, \quad(\ell \leqslant 0.1 t)
$$

for the loops in radiation dominated era, and

$$
n_{\mathrm{CS}}^{r, m}(\ell, t)=\frac{0.18 t_{\mathrm{eq}}^{1 / 2}}{t^{2}(\ell+\Gamma G \mu t)^{5 / 2}}, \quad\left(\ell \leqslant 0.09 t_{\mathrm{eq}}-\Gamma G \mu t\right)
$$

for the loops produced in radiation dominated era but survive until matter domination, where $\Gamma=50$ [65], and $t_{\mathrm{eq}}=2.25 \times 10^{36} \mathrm{GeV}^{-1}$ is the matter-radiation equality time. In the matter dominated era, the loop number density is

$$
n_{\mathrm{CS}}^{m}(\ell, t)=\frac{0.27-0.45(\ell / t)^{0.31}}{t^{2}(\ell+\Gamma G \mu t)^{2}}, \quad(\ell \leqslant 0.18 t) .
$$

Up to now, the GW spectrum is calculable for a given $G \mu$. 


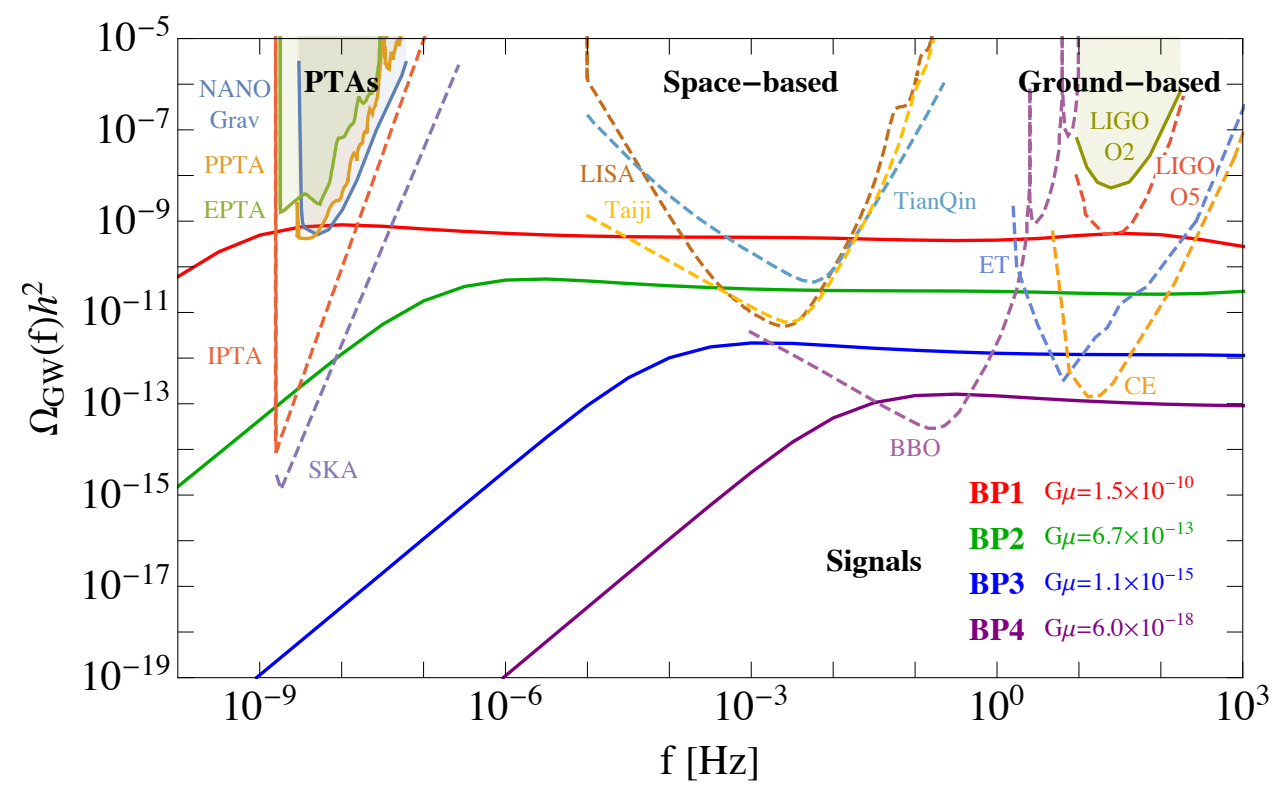

Figure 2. The GW signals from the BPs in table 2, and the relevant sensitivity curves of current or future GW detectors. The existing constraints and projected sensitivities are shown as shaded regions and dashed lines, respectively.

Using eq. (4.1), we evaluate the GW signals for the four BPs in table 2, and plot them as red, green, blue and purple lines in figure 2. Due to the continuous GW emission, the signal spectra are flat in a large frequency range. The sensitivity curves of current and future GW detectors are also plotted in the figure, including the pulsar timing arrays (PTAs) NANOGrav [68-71], PPTA [72, 73], EPTA [74-76], IPTA [77-80] and SKA [81-83], the space-based laser interferometers LISA [84], BBO [85], TianQin [86-88] and Taiji [89, 90], and the ground-based interferometers LIGO [91, 92], CE [93] and ET [94-96]. ${ }^{5}$ The existing constraints are shown as shaded regions, while the projected sensitivities are plotted as dashed lines.

The current searches for the SGWB of PTAs constrains $G \mu<10^{-11}[98,99]$ which requires the $M_{\nu_{R}} / \lambda_{R}<3.2 \times 10^{13} \mathrm{GeV}$ according to eq. (4.1). In figure 2, we can see that $\mathrm{BP} 1$ (with $M_{\nu_{R}}=1.5 \times 10^{13} \mathrm{GeV}$ ) is already within the reach of the PPTA and NANOGrav experiments, and the null results of these observations suggest that the BP1 is excluded; but a DM with mass slightly lower than BP1 is still allowed and can be tested in the near future. Interestingly, recently the NANOGrav collaboration reported a common-spectrum process based on the 12.5-yr data set [100], which might be a hint for the stochastic GW background from cosmic string [101-103]. However, there is some discrepancy between the results of the NANOGrav and PPTA collaborations, and we still have to wait for the future data of the PTA experiments or even the crosscheck from the space- and ground-based detectors to reveal the origin of the excess. The GW signals from BP2 and BP3 can be accessed by a few future detectors, while the GWs from BP4 (with $M_{\nu_{R}}=3.0 \times 10^{9} \mathrm{GeV}$ )

\footnotetext{
${ }^{5}$ The sensitivity curves shown here are strain noise spectra. For the corresponding power-law-integrated sensitivity curves and peak-integrated sensitivity curves, see ref. [97] and the references therein.
} 
can be reached only by $\mathrm{BBO}$ and $\mathrm{CE}$. Therefore, the cosmic strings induced GWs can probe our scenario for RHN DM mass between $\sim 10^{9} \mathrm{GeV}$ and $\sim 10^{13} \mathrm{GeV}$. We also note that there are still considerable uncertainties in calculating the cosmic string GWs, and the expected reach for DM mass range might vary when more accurate treatments are available. $^{6}$

\section{Conclusion}

In this article, we have realized the zombie collision DM mechanism in an extended $\mathrm{U}(1)_{B-L}$ model, showing it allows RHN DM mass up to $10^{13} \mathrm{GeV}$ with a moderate interaction coupling. Such a superheavy DM scenario benefits from both the exponentially annihilation rate due to the lighter sterile neutrino, and the late time entropy production from the sterile neutrino decay.

As the DM mass significantly exceeds the sensitivity regions of the traditional DM detection experiments, we propose to probe the zombie collision DM scenario via GW astronomy by detecting the GW signals from the cosmic strings formed when the $\mathrm{U}(1)_{B-L}$ breaking. Calculations show that RHN DM mass between $\mathcal{O}\left(10^{9} \mathrm{GeV}\right)$ and $\mathcal{O}\left(10^{13} \mathrm{GeV}\right)$ can be probed by future GW experiments; especially, for DM with mass $\sim 10^{13} \mathrm{GeV}$, the signal might already be reached by the recent NANOGrav results, but more data are needed to clarify this.

\section{Acknowledgments}

We would like to thank Xucheng Gan, Huai-Ke Guo, Yi-Lei Tang, Daniele Teresi, ShaoJiang Wang and Bin Zhu for useful discussions. Ligong Bian was supported by the National Natural Science Foundation of China under the grants Nos.12075041, 12047564, and the Fundamental Research Funds for the Central Universities of China (No. 2021CDJQY011 and No. 2020CDJQY-Z003), and Chongqing Natural Science Foundation (Grants No.cstc2020jcyj-msxmX0814). Xuewen Liu was supported by the National Natural Science Foundation of China under the Grants No. 12005180, and by the Natural Science Foundation of Shandong Province under the Grant No. ZR2020QA083. KPX is supported by Grant Korea NRF-2019R1C1C1010050.

\section{A The interaction rates}

The interaction rates in section 3 are defined as

$$
\begin{aligned}
\gamma_{a b \rightarrow c d} \equiv\left\langle\sigma_{a b \rightarrow c d} v\right\rangle n_{a}^{\mathrm{eq}} n_{b}^{\mathrm{eq}} & =\frac{M_{\nu_{R}}}{64 \pi^{4} z} \int_{s_{\min }}^{\infty} d s \hat{\sigma}_{a b \rightarrow c d}(s) \sqrt{s} K_{1}\left(\frac{\sqrt{s}}{M_{\nu_{R}}} z\right) \\
& =\frac{M_{\nu_{R}}^{4}}{64 \pi^{4} z} \int_{x_{\min }}^{\infty} d x \hat{\sigma}_{a b \rightarrow c d}\left(M_{\nu_{R}}^{2} x\right) \sqrt{x} K_{1}(z \sqrt{x}),
\end{aligned}
$$

\footnotetext{
${ }^{6}$ For example, the LIGO-Virgo O3 data can exclude $G \mu \gtrsim 4 \times 10^{-15}$ for some specific string network models [104], much stronger than the results presented in figure 2.
} 
where $K_{1}$ is the modified Bessel function of the first kind, $s_{\min }=\max \left\{\left(M_{a}+M_{b}\right)^{2},\left(M_{c}+\right.\right.$ $\left.\left.M_{d}\right)^{2}\right\}, x=s / M_{\nu_{R}}^{2}$, and the reduced cross section is

$$
\hat{\sigma}_{a b \rightarrow c d}(s) \equiv 2 \sigma_{a b \rightarrow c d}(s) \cdot s \cdot\left[1-2\left(\frac{M_{a}^{2}}{s}+\frac{M_{b}^{2}}{s}\right)+\left(\frac{M_{a}^{2}}{s}-\frac{M_{b}^{2}}{s}\right)^{2}\right],
$$

where $\sigma_{a b \rightarrow c d}$ is the Lorentz invariant cross section, which is a function of $s$. All initial and final state spin and internal degrees of freedom have been summed up. Our convention of $\gamma_{a b \rightarrow c d}$ is the same as ref. [105].

Since the explicit expressions for the interaction rates in section 3 are too tedious to be shown in the article, below we only list the amplitude squares for the corresponding processes in our mechanism. With the amplitude squares in hand, one can easily derives the cross sections and interaction rates. Assume the collision to be $a\left(p_{1}\right) b\left(p_{2}\right) \rightarrow c\left(p_{3}\right) d\left(p_{4}\right)$, and define the Mandelstam variables as $s=\left(p_{1}+p_{2}\right)^{2}$ and $t=\left(p_{3}-p_{1}\right)^{2}$, for the zombie collisions we have

$$
\begin{aligned}
& \sum_{\text {spins }}\left|i \mathcal{M}_{\nu_{R} \psi \rightarrow \psi \psi}\right|^{2}=\lambda_{1}^{2} \lambda_{2}^{2}\left(M_{S}^{2}-t\right)^{-2}\left(s+t-3 M_{\psi}^{2}+M_{S}^{2}-M_{\nu_{R}}^{2}\right)^{-2} \times \\
& \quad\left\{\left(M_{S}^{2}-t\right)\left(2 M_{\psi}^{2}-s-t\right)\left(M_{\psi}^{2}-M_{\nu_{R}}^{2}+s+t\right)\left(s+3 t-3 M_{\psi}^{2}-M_{S}^{2}-M_{\nu_{R}}^{2}\right)\right. \\
& \quad+\left[\left(4 M_{\psi}^{2}-t\right)\left(M_{\psi}^{2}+M_{\nu_{R}}^{2}-t\right)\left(2 s+3 t-6 M_{\psi}^{2}+M_{S}^{2}-2 M_{\nu_{R}}^{2}\right)\right. \\
& \left.\left.\quad+\left(4 M_{\psi}^{2}-s\right)\left(M_{S}^{2}-t\right)\left(M_{\psi}^{2}+M_{\nu_{R}}^{2}-s\right)\right]\left(s+t-3 M_{\psi}^{2}+M_{S}^{2}-M_{\nu_{R}}^{2}\right)\right\}
\end{aligned}
$$

and

$$
\begin{aligned}
\sum_{\text {spins }}\left|i \mathcal{M}_{\nu_{R} \psi \rightarrow \psi \bar{\psi}}\right|^{2}= & \lambda_{1}^{2} \lambda_{2}^{2}\left(M_{S}^{2}-t\right)^{-2}\left[\left(s-M_{S}^{2}\right)^{2}+M_{S}^{2} \Gamma_{S}^{2}\right]^{-1} \times \\
& \left\{\left(4 M_{\psi}^{2}-t\right)\left(M_{\psi}^{2}+M_{\nu_{R}}^{2}-t\right)\left(2 \Gamma_{S}^{2} M_{S}^{2}+\left(M_{S}^{2}-s\right)\left(M_{S}^{2}-2 s+t\right)\right)\right. \\
& +\left(t-M_{S}^{2}\right)\left[\left(s-4 M_{\psi}^{2}\right)\left(M_{\psi}^{2}+M_{\nu_{R}}^{2}-s\right)\left(M_{S}^{2}+s-2 t\right)\right. \\
& \left.\left.+\left(s-M_{S}^{2}\right)\left(s+t-2 M_{\psi}^{2}\right)\left(s+t+M_{\psi}^{2}-M_{\nu_{R}}^{2}\right)\right]\right\} .
\end{aligned}
$$

Note that in the process $\nu_{R} \psi \rightarrow \psi \bar{\psi}$, the mediator $S$ can be in the $s$-channel, thus its width should be included to make the integral in eq. (A.1) converge.

For the pair annihilation of $\nu_{R} \nu_{R} \rightarrow \psi \bar{\psi}$, there are both contributions from the Yukawa interactions and $\mathrm{U}(1)_{B-L}$ gauge interactions,

$$
\begin{aligned}
\sum_{\text {spins }}\left|i \mathcal{M}_{\nu_{R} \nu_{R} \rightarrow \psi \bar{\psi}}\right|^{2}= & \lambda_{1}^{4}\left\{\frac{2 M_{\nu_{R}}^{2}\left(s-2 M_{\psi}^{2}\right)}{\left(t-M_{S}^{2}\right)\left(s+t-2 M_{\psi}^{2}+M_{S}^{2}-2 M_{\nu_{R}}^{2}\right)}\right. \\
& \left.+\frac{\left(M_{\psi}^{2}+M_{\nu_{R}}^{2}-s-t\right)^{2}}{\left(s+t-2 M_{\psi}^{2}+M_{S}^{2}-2 M_{\nu_{R}}^{2}\right)^{2}}+\frac{\left(M_{\psi}^{2}+M_{\nu_{R}}^{2}-t\right)^{2}}{\left(M_{S}^{2}-t\right)^{2}}\right\} \\
& +8 g_{B-L}^{4} \frac{2 M_{\psi}^{4}-4 M_{\psi}^{2}\left(M_{\nu_{R}}^{2}+t\right)+2 M_{\nu_{R}}^{4}-4 M_{\nu_{R}}^{2}(s+t)+s^{2}+2 s t+2 t^{2}}{\left(s-M_{Z^{\prime}}^{2}\right)^{2}+M_{Z^{\prime}}^{2} \Gamma_{Z^{\prime}}^{2}}
\end{aligned}
$$


while for $\nu_{R} \nu_{R} \rightarrow \psi \psi$, only Yukawa interactions contribute,

$$
\begin{aligned}
& \sum_{\text {spins }}\left|i \mathcal{M}_{\nu_{R} \nu_{R} \rightarrow \psi \psi}\right|^{2}=\lambda_{1}^{4}\left(M_{S}^{2}-t\right)^{-2}\left(s+t-2 M_{\psi}^{2}+M_{S}^{2}-2 M_{\nu_{R}}^{2}\right)^{-2} \times \\
& \qquad\left(s+2 t-2 M_{\psi}^{2}-2 M_{\nu_{R}}^{2}\right)^{2}\left[t\left(s-2\left(M_{\psi}^{2}+M_{\nu_{R}}^{2}\right)\right)+\left(M_{\psi}^{2}+M_{\nu_{R}}^{2}\right)^{2}-s M_{S}^{2}+t^{2}\right] \\
& \left.\quad+\left(s-2 M_{\psi}^{2}\right)\left(M_{S}^{2}-t\right)\left(s-2 M_{\nu_{R}}^{2}\right)\left(s+t-2 M_{\psi}^{2}+M_{S}^{2}-2 M_{\nu_{R}}^{2}\right)\right\} .
\end{aligned}
$$

Finally, the $Z^{\prime}$ mediated annihilation to the SM fermions reads

$$
\begin{gathered}
\sum_{\text {spins }}\left|i \mathcal{M}_{\nu_{R} \nu_{R} \rightarrow f \bar{f}}\right|^{2}=\frac{13}{2} \times 8 g_{B-L}^{4} \frac{\left(s+t-2 M_{\nu_{R}}^{2}\right)^{2}+t^{2}-2 M_{\nu_{R}}^{4},}{\left(s-M_{Z^{\prime}}^{2}\right)^{2}+M_{Z^{\prime}}^{2} \Gamma_{Z^{\prime}}^{2}}, \\
\sum_{\text {spins }}\left|i \mathcal{M}_{\psi \bar{\psi} \rightarrow f \bar{f}}\right|^{2}=\frac{13}{2} \times 8 g_{B-L}^{4} \frac{(s+t)^{2}+\left(2 M_{\psi}^{2}-t\right)^{2}-2 M_{\psi}^{4}}{\left(s-M_{Z^{\prime}}^{2}\right)^{2}+M_{Z^{\prime}}^{2} \Gamma_{Z^{\prime}}^{2}} .
\end{gathered}
$$

Here the factor $13 / 2$ comes from the summation of SM fermionic degrees of freedom.

Open Access. This article is distributed under the terms of the Creative Commons Attribution License (CC-BY 4.0), which permits any use, distribution and reproduction in any medium, provided the original author(s) and source are credited.

\section{References}

[1] B.W. Lee and S. Weinberg, Cosmological Lower Bound on Heavy Neutrino Masses, Phys. Rev. Lett. 39 (1977) 165 [INSPIRE].

[2] E.W. Kolb and M.S. Turner, Frontiers in Physics. Vol. 69: The Early Universe, CRC Press, Boca Raton U.S.A. (1990).

[3] G. Bertone, D. Hooper and J. Silk, Particle dark matter: Evidence, candidates and constraints, Phys. Rept. 405 (2005) 279 [hep-ph/0404175] [INSPIRE].

[4] M. Lisanti, Lectures on Dark Matter Physics, in Theoretical Advanced Study Institute in Elementary Particle Physics: New Frontiers in Fields and Strings, Boulder U.S.A. (2015), pg. 399 [arXiv: 1603.03797] [INSPIRE].

[5] Planck collaboration, Planck 2018 results. VI. Cosmological parameters, Astron. Astrophys. 641 (2020) A6 [Erratum ibid. 652 (2021) C4] [arXiv:1807.06209] [InSPIRE].

[6] Particle Data Group collaboration, Review of Particle Physics, PTEP 2020 (2020) 083C01 [INSPIRE].

[7] M. Schumann, Direct Detection of WIMP Dark Matter: Concepts and Status, J. Phys. G 46 (2019) 103003 [arXiv: 1903.03026] [INSPIRE].

[8] J.M. Gaskins, A review of indirect searches for particle dark matter, Contemp. Phys. 57 (2016) 496 [arXiv: 1604.00014].

[9] R. Kitano, H. Ooguri and Y. Ookouchi, Supersymmetry Breaking and Gauge Mediation, Ann. Rev. Nucl. Part. Sci. 60 (2010) 491 [arXiv:1001.4535] [InSPIRE].

[10] K. Griest and M. Kamionkowski, Unitarity Limits on the Mass and Radius of Dark Matter Particles, Phys. Rev. Lett. 64 (1990) 615 [InSPIRE]. 
[11] E.W. Kolb, D.J.H. Chung and A. Riotto, WIMPzillas!, AIP Conf. Proc. 484 (1999) 91 [hep-ph/9810361] [INSPIRE].

[12] L. Hui and E.D. Stewart, Superheavy dark matter from thermal inflation, Phys. Rev. D 60 (1999) 023518 [hep-ph/9812345] [INSPIRE].

[13] D.J.H. Chung, E.W. Kolb and A. Riotto, Production of massive particles during reheating, Phys. Rev. D 60 (1999) 063504 [hep-ph/9809453] [INSPIRE].

[14] D.J.H. Chung, P. Crotty, E.W. Kolb and A. Riotto, On the Gravitational Production of Superheavy Dark Matter, Phys. Rev. D 64 (2001) 043503 [hep-ph/0104100] [InSPIRE].

[15] K. Harigaya, M. Kawasaki, K. Mukaida and M. Yamada, Dark Matter Production in Late Time Reheating, Phys. Rev. D 89 (2014) 083532 [arXiv:1402.2846] [InSPIRE].

[16] H. Davoudiasl, D. Hooper and S.D. McDermott, Inflatable Dark Matter, Phys. Rev. Lett. 116 (2016) 031303 [arXiv: 1507.08660] [INSPIRE].

[17] L. Randall, J. Scholtz and J. Unwin, Flooded Dark Matter and S Level Rise, JHEP 03 (2016) 011 [arXiv: 1509.08477] [INSPIRE].

[18] K. Harigaya, T. Lin and H.K. Lou, GUTzilla Dark Matter, JHEP 09 (2016) 014 [arXiv: 1606. 00923] [INSPIRE].

[19] A. Berlin, D. Hooper and G. Krnjaic, PeV-Scale Dark Matter as a Thermal Relic of a Decoupled Sector, Phys. Lett. B $\mathbf{7 6 0}$ (2016) 106 [arXiv:1602.08490] [INSPIRE].

[20] A. Berlin, D. Hooper and G. Krnjaic, Thermal Dark Matter From A Highly Decoupled Sector, Phys. Rev. D 94 (2016) 095019 [arXiv: 1609.02555] [InSPIRE].

[21] J. Bramante and J. Unwin, Superheavy Thermal Dark Matter and Primordial Asymmetries, JHEP 02 (2017) 119 [arXiv:1701.05859] [inSPIRE].

[22] S. Hamdan and J. Unwin, Dark Matter Freeze-out During Matter Domination, Mod. Phys. Lett. A 33 (2018) 1850181 [arXiv:1710.03758] [INSPIRE].

[23] M. Cirelli, Y. Gouttenoire, K. Petraki and F. Sala, Homeopathic Dark Matter, or how diluted heavy substances produce high energy cosmic rays, JCAP 02 (2019) 014 [arXiv: 1811.03608] [INSPIRE].

[24] E. Babichev, D. Gorbunov and S. Ramazanov, New mechanism of producing superheavy Dark Matter, Phys. Lett. B $\mathbf{7 9 4}$ (2019) 69 [arXiv:1812.03516] [InSPIRE].

[25] S. Hashiba and J. Yokoyama, Gravitational particle creation for dark matter and reheating, Phys. Rev. D 99 (2019) 043008 [arXiv:1812.10032] [INSPIRE].

[26] D. Hooper, G. Krnjaic and S.D. McDermott, Dark Radiation and Superheavy Dark Matter from Black Hole Domination, JHEP 08 (2019) 001 [arXiv:1905.01301] [INSPIRE].

[27] H. Davoudiasl and G. Mohlabeng, Getting a THUMP from a WIMP, JHEP 04 (2020) 177 [arXiv: 1912.05572] [INSPIRE].

[28] P. Chanda, S. Hamdan and J. Unwin, Reviving $Z$ and Higgs Mediated Dark Matter Models in Matter Dominated Freeze-out, JCAP 01 (2020) 034 [arXiv: 1911.02616] [INSPIRE].

[29] M.J. Baker, J. Kopp and A.J. Long, Filtered Dark Matter at a First Order Phase Transition, Phys. Rev. Lett. 125 (2020) 151102 [arXiv: 1912.02830] [INSPIRE].

[30] D. Chway, T.H. Jung and C.S. Shin, Dark matter filtering-out effect during a first-order phase transition, Phys. Rev. D 101 (2020) 095019 [arXiv:1912.04238] [INSPIRE]. 
[31] D. Marfatia and P.-Y. Tseng, Gravitational wave signals of dark matter freeze-out, JHEP 02 (2021) 022 [arXiv: 2006. 07313] [INSPIRE].

[32] A. Azatov, M. Vanvlasselaer and W. Yin, Dark Matter production from relativistic bubble walls, JHEP 03 (2021) 288 [arXiv:2101.05721] [INSPIRE].

[33] A. Berlin, WIMPs with GUTs: Dark Matter Coannihilation with a Lighter Species, Phys. Rev. Lett. 119 (2017) 121801 [arXiv:1704.08256] [INSPIRE].

[34] E.D. Kramer, E. Kuflik, N. Levi, N.J. Outmezguine and J.T. Ruderman, Heavy Thermal Dark Matter from a New Collision Mechanism, Phys. Rev. Lett. 126 (2021) 081802 [arXiv: 2003.04900] [INSPIRE].

[35] A. Davidson, $B-L$ as the fourth color within an $\mathrm{SU}(2)_{L} \times \mathrm{U}(1)_{R} \times \mathrm{U}(1)$ model, Phys. Rev. $D 20$ (1979) 776 [INSPIRE].

[36] R.E. Marshak and R.N. Mohapatra, Quark-Lepton Symmetry and B-L as the U(1) Generator of the Electroweak Symmetry Group, Phys. Lett. B 91 (1980) 222 [INSPIRE].

[37] R.N. Mohapatra and R.E. Marshak, Local B-L Symmetry of Electroweak Interactions, Majorana Neutrinos and Neutron Oscillations, Phys. Rev. Lett. 44 (1980) 1316 [Erratum ibid. 44 (1980) 1643] [INSPIRE].

[38] A. Davidson and K.C. Wali, Universal Seesaw Mechanism?, Phys. Rev. Lett. 59 (1987) 393 [INSPIRE].

[39] P. Minkowski, $\mu \rightarrow$ ey at a Rate of One Out of $10^{9}$ Muon Decays?, Phys. Lett. B 67 (1977) 421 [INSPIRE].

[40] M. Fukugita and T. Yanagida, Baryogenesis Without Grand Unification, Phys. Lett. B 174 (1986) 45 [INSPIRE].

[41] KATRIN collaboration, Improved Upper Limit on the Neutrino Mass from a Direct Kinematic Method by KATRIN, Phys. Rev. Lett. 123 (2019) 221802 [arXiv:1909.06048] [INSPIRE].

[42] N. Okada and O. Seto, Higgs portal dark matter in the minimal gauged $\mathrm{U}(1)_{B-L}$ model, Phys. Rev. D 82 (2010) 023507 [arXiv: 1002.2525] [INSPIRE].

[43] N. Okada and Y. Orikasa, Dark matter in the classically conformal B-L model, Phys. Rev. D 85 (2012) 115006 [arXiv:1202.1405] [INSPIRE].

[44] N. Okada and S. Okada, $Z^{\prime}$-portal right-handed neutrino dark matter in the minimal $\mathrm{U}(1)_{X}$ extended Standard Model, Phys. Rev. D 95 (2017) 035025 [arXiv:1611.02672] [InSPIRE].

[45] N. Okada and S. Okada, $Z_{B L}^{\prime}$ portal dark matter and LHC Run-2 results, Phys. Rev. D 93 (2016) 075003 [arXiv: 1601.07526] [INSPIRE].

[46] S. Okada, $Z^{\prime}$ Portal Dark Matter in the Minimal B - L Model, Adv. High Energy Phys. 2018 (2018) 5340935 [arXiv: 1803.06793] [INSPIRE].

[47] D. Borah, D. Nanda, N. Narendra and N. Sahu, Right-handed neutrino dark matter with radiative neutrino mass in gauged B - L model, Nucl. Phys. B 950 (2020) 114841 [arXiv:1810.12920].

[48] Z.-z. Xing and Z.-h. Zhao, The minimal seesaw and leptogenesis models, Rept. Prog. Phys. 84 (2021) 066201 [arXiv:2008.12090] [INSPIRE]. 
[49] L. Bian, W. Cheng, H.-K. Guo and Y. Zhang, Cosmological implications of a B - L charged hidden scalar: leptogenesis and gravitational waves, Chin. Phys. C 45 (2021) 113104 [arXiv: 1907.13589] [INSPIRE].

[50] M. Cirelli, E. Moulin, P. Panci, P.D. Serpico and A. Viana, Gamma ray constraints on Decaying Dark Matter, Phys. Rev. D 86 (2012) 083506 [arXiv:1205.5283] [inSPIRE].

[51] R. Essig, E. Kuflik, S.D. McDermott, T. Volansky and K.M. Zurek, Constraining Light Dark Matter with Diffuse X-Ray and Gamma-Ray Observations, JHEP 11 (2013) 193 [arXiv: 1309.4091] [INSPIRE].

[52] C. Blanco and D. Hooper, Constraints on Decaying Dark Matter from the Isotropic Gamma-Ray Background, JCAP 03 (2019) 019 [arXiv:1811.05988] [INSPIRE].

[53] E.W. Kolb and M.S. Turner, The Early Universe, Nature 294 (1981) 521 [InSPIRE].

[54] A. Alloul, N.D. Christensen, C. Degrande, C. Duhr and B. Fuks, FeynRules $2.0-A$ complete toolbox for tree-level phenomenology, Comput. Phys. Commun. 185 (2014) 2250 [arXiv:1310.1921] [INSPIRE].

[55] J. Alwall et al., The automated computation of tree-level and next-to-leading order differential cross sections, and their matching to parton shower simulations, JHEP $\mathbf{0 7}$ (2014) 079 [arXiv: 1405.0301] [INSPIRE].

[56] W. Buchmüller, V. Domcke, K. Kamada and K. Schmitz, The Gravitational Wave Spectrum from Cosmological B - L Breaking, JCAP 10 (2013) 003 [arXiv: 1305.3392] [InSPIRE].

[57] J.A. Dror, T. Hiramatsu, K. Kohri, H. Murayama and G. White, Testing the Seesaw Mechanism and Leptogenesis with Gravitational Waves, Phys. Rev. Lett. 124 (2020) 041804 [arXiv: 1908.03227] [INSPIRE].

[58] P. Auclair et al., Probing the gravitational wave background from cosmic strings with LISA, JCAP 04 (2020) 034 [arXiv: 1909. 00819] [INSPIRE].

[59] B. Fornal and B. Shams Es Haghi, Baryon and Lepton Number Violation from Gravitational Waves, Phys. Rev. D 102 (2020) 115037 [arXiv: 2008.05111] [InSPIRE].

[60] R. Samanta and S. Datta, Gravitational wave complementarity and impact of NANOGrav data on gravitational leptogenesis, JHEP 05 (2021) 211 [arXiv:2009.13452] [INSPIRE].

[61] M.A. Masoud, M.U. Rehman and Q. Shafi, Sneutrino tribrid inflation, metastable cosmic strings and gravitational waves, JCAP 11 (2021) 022 [arXiv:2107.09689] [INSPIRE].

[62] W. Buchmüller, V. Domcke and K. Schmitz, Stochastic gravitational-wave background from metastable cosmic strings, arXiv:2107.04578 [INSPIRE].

[63] H.B. Nielsen and P. Olesen, Vortex Line Models for Dual Strings, Nucl. Phys. B 61 (1973) 45 [INSPIRE].

[64] T. Vachaspati and A. Vilenkin, Gravitational Radiation from Cosmic Strings, Phys. Rev. D 31 (1985) 3052 [INSPIRE].

[65] J.J. Blanco-Pillado and K.D. Olum, Stochastic gravitational wave background from smoothed cosmic string loops, Phys. Rev. D 96 (2017) 104046 [arXiv:1709.02693] [InSPIRE].

[66] P. Binetruy, A. Bohe, C. Caprini and J.-F. Dufaux, Cosmological Backgrounds of Gravitational Waves and eLISA/NGO: Phase Transitions, Cosmic Strings and Other Sources, JCAP 06 (2012) 027 [arXiv: 1201.0983] [INSPIRE]. 
[67] J.J. Blanco-Pillado, K.D. Olum and B. Shlaer, The number of cosmic string loops, Phys. Rev. D 89 (2014) 023512 [arXiv: 1309.6637] [INSPIRE].

[68] M.A. McLaughlin, The North American Nanohertz Observatory for Gravitational Waves, Class. Quant. Grav. 30 (2013) 224008 [arXiv:1310.0758] [inSPIRE].

[69] NANOGRAV collaboration, The NANOGrav 11-year Data Set: Pulsar-timing Constraints On The Stochastic Gravitational-wave Background, Astrophys. J. 859 (2018) 47 [arXiv: 1801.02617] [INSPIRE].

[70] K. Aggarwal et al., The NANOGrav 11-Year Data Set: Limits on Gravitational Waves from Individual Supermassive Black Hole Binaries, Astrophys. J. 880 (2019) 2 [arXiv: 1812.11585] [INSPIRE].

[71] A. Brazier et al., The NANOGrav Program for Gravitational Waves and Fundamental Physics, arXiv: 1908.05356 [INSPIRE].

[72] R.N. Manchester et al., The Parkes Pulsar Timing Array Project, Publ. Astron. Soc. Austral. 30 (2013) 17 [arXiv:1210.6130] [INSPIRE].

[73] R.M. Shannon et al., Gravitational waves from binary supermassive black holes missing in pulsar observations, Science 349 (2015) 1522 [arXiv: 1509.07320] [INSPIRE].

[74] M. Krämer and D.J. Champion, The European Pulsar Timing Array and the Large European Array for Pulsars, Class. Quant. Grav. 30 (2013) 224009 [InSPIRE].

[75] L. Lentati et al., European Pulsar Timing Array Limits On An Isotropic Stochastic Gravitational-Wave Background, Mon. Not. Roy. Astron. Soc. 453 (2015) 2576 [arXiv: 1504.03692] [INSPIRE].

[76] S. Babak et al., European Pulsar Timing Array Limits on Continuous Gravitational Waves from Individual Supermassive Black Hole Binaries, Mon. Not. Roy. Astron. Soc. 455 (2016) 1665 [arXiv: 1509.02165] [INSPIRE].

[77] G. Hobbs et al., The international pulsar timing array project: using pulsars as a gravitational wave detector, Class. Quant. Grav. 27 (2010) 084013 [arXiv:0911.5206] [INSPIRE].

[78] R.N. Manchester, The International Pulsar Timing Array, Class. Quant. Grav. 30 (2013) 224010 [arXiv: 1309.7392] [INSPIRE].

[79] J.P.W. Verbiest et al., The International Pulsar Timing Array: First Data Release, Mon. Not. Roy. Astron. Soc. 458 (2016) 1267 [arXiv: 1602.03640] [INSPIRE].

[80] J.S. Hazboun, C.M.F. Mingarelli and K. Lee, The Second International Pulsar Timing Array Mock Data Challenge, arXiv:1810.10527 [INSPIRE].

[81] C.L. Carilli and S. Rawlings, Science with the Square Kilometer Array: Motivation, key science projects, standards and assumptions, New Astron. Rev. 48 (2004) 979 [astro-ph/0409274] [INSPIRE].

[82] G. Janssen et al., Gravitational wave astronomy with the SKA, PoS AASKA14 (2015) 037 [arXiv: 1501.00127] [INSPIRE].

[83] A. Weltman et al., Fundamental physics with the Square Kilometre Array, Publ. Astron. Soc. Austral. 37 (2020) e002 [arXiv: 1810.02680] [INSPIRE].

[84] LISA collaboration, Laser Interferometer Space Antenna, arXiv:1702.00786 [InSPIRE]. 
[85] J. Crowder and N.J. Cornish, Beyond LISA: Exploring future gravitational wave missions, Phys. Rev. D 72 (2005) 083005 [gr-qc/0506015] [inSPIRE].

[86] TIAnQIN collaboration, TianQin: a space-borne gravitational wave detector, Class. Quant. Grav. 33 (2016) 035010 [arXiv: 1512.02076] [INSPIRE].

[87] Y.-M. Hu, J. Mei and J. Luo, Science prospects for space-borne gravitational-wave missions, Natl. Sci. Rev. 4 (2017) 683 [InSPIRE].

[88] TianQin collaboration, The TianQin project: current progress on science and technology, arXiv:2008.10332 [INSPIRE].

[89] W.-R. Hu and Y.-L. Wu, The Taiji Program in Space for gravitational wave physics and the nature of gravity, Natl. Sci. Rev. 4 (2017) 685 [INSPIRE].

[90] W.-H. Ruan, Z.-K. Guo, R.-G. Cai and Y.-Z. Zhang, Taiji program: Gravitational-wave sources, Int. J. Mod. Phys. A 35 (2020) 2050075 [arXiv: 1807.09495] [INSPIRE].

[91] LIGO ScIENTIFIC and Virgo collaborations, Characterization of the LIGO detectors during their sixth science run, Class. Quant. Grav. 32 (2015) 115012 [arXiv:1410.7764] [INSPIRE].

[92] LIGO SCIENTIFIC and VIRGo collaborations, Search for the isotropic stochastic background using data from Advanced LIGO's second observing run, Phys. Rev. D 100 (2019) 061101 [arXiv: 1903. 02886] [INSPIRE].

[93] D. Reitze et al., Cosmic Explorer: The U.S. Contribution to Gravitational-Wave Astronomy beyond LIGO, Bull. Am. Astron. Soc. 51 (2019) 035 [arXiv:1907.04833] [inSPIRE].

[94] M. Punturo et al., The Einstein Telescope: A third-generation gravitational wave observatory, Class. Quant. Grav. 27 (2010) 194002 [InSPIRE].

[95] S. Hild et al., Sensitivity Studies for Third-Generation Gravitational Wave Observatories, Class. Quant. Grav. 28 (2011) 094013 [arXiv: 1012.0908] [inSPIRE].

[96] B. Sathyaprakash et al., Scientific Objectives of Einstein Telescope, Class. Quant. Grav. 29 (2012) 124013 [Erratum ibid. 30 (2013) 079501] [arXiv: 1206. 0331] [INSPIRE].

[97] K. Schmitz, New Sensitivity Curves for Gravitational-Wave Signals from Cosmological Phase Transitions, JHEP 01 (2021) 097 [arXiv:2002.04615] [INSPIRE].

[98] C. Ringeval and T. Suyama, Stochastic gravitational waves from cosmic string loops in scaling, JCAP 12 (2017) 027 [arXiv:1709.03845] [INSPIRE].

[99] J.J. Blanco-Pillado, K.D. Olum and X. Siemens, New limits on cosmic strings from gravitational wave observation, Phys. Lett. B 778 (2018) 392 [arXiv:1709.02434] [INSPIRE].

[100] NANOGRAV collaboration, The NANOGrav 12.5 yr Data Set: Search for an Isotropic Stochastic Gravitational-wave Background, Astrophys. J. Lett. 905 (2020) L34 [arXiv: 2009. 04496] [INSPIRE].

[101] J. Ellis and M. Lewicki, Cosmic String Interpretation of NANOGrav Pulsar Timing Data, Phys. Rev. Lett. 126 (2021) 041304 [arXiv:2009.06555] [INSPIRE].

[102] L. Bian, R.-G. Cai, J. Liu, X.-Y. Yang and R. Zhou, Evidence for different gravitational-wave sources in the NANOGrav dataset, Phys. Rev. D 103 (2021) L081301 [arXiv:2009.13893] [INSPIRE]. 
[103] S. Blasi, V. Brdar and K. Schmitz, Has NANOGrav found first evidence for cosmic strings?, Phys. Rev. Lett. 126 (2021) 041305 [arXiv: 2009. 06607] [INSPIRE].

[104] LIGO Scientific, Virgo and KAGRA collaborations, Constraints on Cosmic Strings Using Data from the Third Advanced LIGO-Virgo Observing Run, Phys. Rev. Lett. 126 (2021) 241102 [arXiv : 2101.12248] [inSPIRE].

[105] G.F. Giudice, A. Notari, M. Raidal, A. Riotto and A. Strumia, Towards a complete theory of thermal leptogenesis in the SM and MSSM, Nucl. Phys. B 685 (2004) 89 [hep-ph/0310123] [INSPIRE]. 